\title{
A Hybrid Restoration Approach of Defocused Image Using MGAM and Inverse Filtering
}

\author{
Fenglan $\mathrm{Li}^{1}$, Liyun $\mathrm{Su}^{2}$ \\ ${ }^{1}$ Library, Chongqing University of Technology, Chongqing, 400054 China \\ lifenglan@cqut.edu.cn \\ Yun Jiang ${ }^{1}$, Min Sun ${ }^{2}$ \\ ${ }^{2}$ School of Mathematics and Statistics, Chongqing University of Technology, Chongqing, 400054 China \\ cloudhopping@163.com
}

\begin{abstract}
A novel hybrid restoration scheme of defocused image is presented, which uses multivariate generalized additive model (MGAM) which is a nonparametric statistical regression model with no curse of dimensionality and inverse filtering (InvF). In this algorithm, firstly the five features of wavelet domain in defocused digital image, which are very stable relationship with the point spread function (PSF) parameter, are extracted by training and fitting a multivariate generalized additive model which is to estimate defocused blurred parameter. After the point spread function parameter is obtained, inverse filtering, which is needed to known the point spread function and a non-blind restoration method, is applied to complete the restoration for getting the true image. Simulated and real blurred images are experimentally illustrated to evaluate performances of the presented method. Results show that the proposed defocused image hybrid restoration technique is effective and robust.
\end{abstract}

Index Terms - Defocused Image Restoration; Wavelet Transform; Multivariate Generalized Additive Model (MGAM); Inverse Filtering (InvF)

\section{INTRODUCTION}

Shooting a real world image with a camera, at least some parts are affected by a blur and noise. Atmospheric turbulence, longer exposures, and so on can blur images, but the exact cause of blurring may be unknown. Restoration in the case of known blur, assuming the linear degradation model, is called linear image restoration and it has been presented extensively in the last three decades giving rise to a variety of solutions [1-3]. In many practical situations, however, the blur is unknown. Hence, both blur identification and image restoration must be performed from the degraded image. Restoration in the case of unknown blur is called blind image restoration [4, 5]. Existing blind restoration methods can be categorized into two main groups: (i) those which estimate the PSF a priori independent of the true image so as to use it later with one of the linear image restoration methods, such as zero sheet separation, generalized cross validation, and maximum likelihood and expectation maximization based on the ARMA image model $[6,7]$, and (ii) those which estimate the PSF and the true image simultaneously, such as nonnegative sand support constraints recursive inverse filtering, maximum likelihood and conjugate gradient minimization, and simulated annealing $[8,9]$. Algorithms belonging to the first class are computationally simple, but they are limited to situations in which the PSF has a special form, and the true image has certain features. The investigated defocused image restoration algorithms by using local polynomial nonparametric regression method belongs to the second class, which are computationally more complex, then it must be used for more general situations. In this paper, a kind of semi-blind image restoration algorithm is proposed in case of known the blur type (defocused blurring) with bivariate generalized nonparametric statistical regression additive model of no curse of dimensionality and good local kernel smoothing technique and classical image restoration method inverse filtering.

In general, discrete bivariate digital image model for a linear degradation caused by blurring and additive normal white noise with mean 0 can be given by the following equation

$$
y(i, j)=h(i, j) * f(i, j)+n(i, j),
$$

where $*$ indicates two-dimensional convolution, $f(i, j)$ represents on original image, $y(i, j)$ is the degraded image, $h(i, j)$ represents the two-dimensional PSF, and $n(i, j)$ is the additive noise. As for defocus blur, PSF is modeled as a uniform intensity distribution within a circular disk, 
$h(i, j)=\left\{\begin{array}{ll}\frac{1}{\pi R^{2}} & \text { if } \sqrt{i^{2}+j^{2}} \leq R \\ 0 & \text { otherwise }\end{array}\right.$,

where disk radius $R$ is the only unknown parameter for this type of blur.

Fourier methods can be used to estimate the defocus parameter $R$ through calculating a ratio of power of high frequencies portion to that of low frequencies portion. However, a main drawback of the method is its bad noise immunity. To solve this problem, literature [11] has proposed a novel algorithm to overcome this shortcoming, the RBF neural network is applied to fit $R$. This scheme has good fitting, but bad prediction. To avoid the weak generalization ability, a more efficient method for estimating parameter $R$ is proposed in this paper. The prediction ability of these two methods is compared with the trained five images. The steps of the presented algorithm in this paper is as follows: Firstly we construct feature vectors of several blurred images with known defocus radius $R$ in wavelet domain of defocused digital image, then a multivariate generalized additive model (MGAM) [12-15] with nonparametric local kernel technique [16-19] is trained using the vector $\mathrm{s}$ as inputs and defocused parameters as outputs. After the model is trained, the new defocused images are applied to the trained model for predicting the parameter $R$. In this paper, inverse filtering ( InvF) [4, $20]$ is adopted to image restoration.

Here the features in wavelet domain of defocused digital image are discussed. The $l$ level wavelet decomposition of the defocused digital image $I$ results in an approximation image $X_{l}$ and three detailed images $H_{l}, V_{l}$, and $D_{l}$ in horizontal, vertical, and diagonal directions respectively. Decomposition into $l$ levels of an original image results in a down sampled image of resolution $2^{l}$ with respect to the image as well as detail images. In order to denote the relationship between wavelet coefficients and defocused radius $R$, we define five variables named $v_{1}, v_{2}, v_{3}, v_{4}$, and $v_{5}$ as:

$$
\left\{\begin{array}{l}
v_{1}=\left|V_{2}\right|_{s} /\left|H_{2}\right|_{s}, \\
v_{2}=\left|H_{2}\right|_{s} /\left|X_{2}\right|_{s}, \\
v_{3}=\left|H_{1}\right|_{s} / \operatorname{mum}\left\{H_{1}\right\}, \\
v_{4}=\left|H_{2}\right|_{s} / \operatorname{mum}\left\{H_{2}\right\}, \\
v_{5}=\left|D_{1}\right|_{s} / \operatorname{mum}\left\{D_{1}\right\}
\end{array}\right.
$$

where $|\cdot|_{\mathrm{s}}$ represents the summation of all coefficients' absolute value, num $\{\cdot\}$ is total number of coefficients.

An original image is blurred artificially by a uniform defocused point spread function (PSF) with parameter $R$ whose value ranging from 1 to 20 . The relationships between $v_{1}, v_{2}, v_{3}, v_{4}, v_{5}$ and $R$ are shown in Fig.1, where the curves are normalized in $[0,1]$ interval. When $R$ increases, $v_{2}, v_{3}, v_{4}$ and $v_{5}$ decrease monotonously.
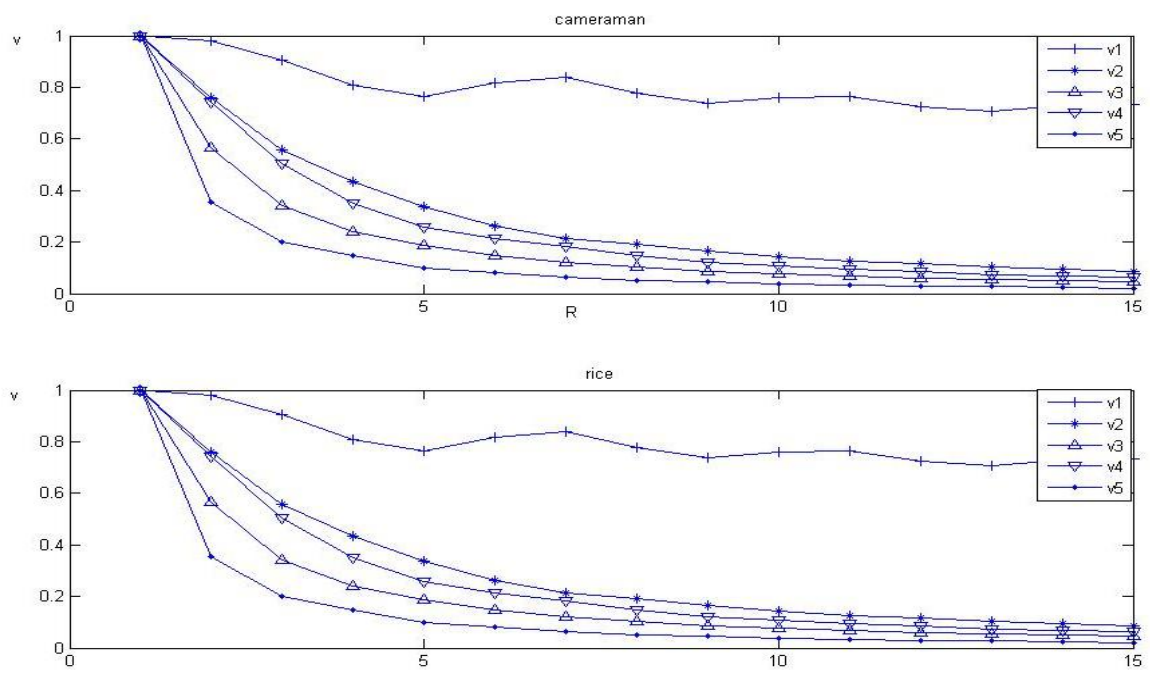

Figure 1.Relationship between $v_{1-5}$ and $R$ 

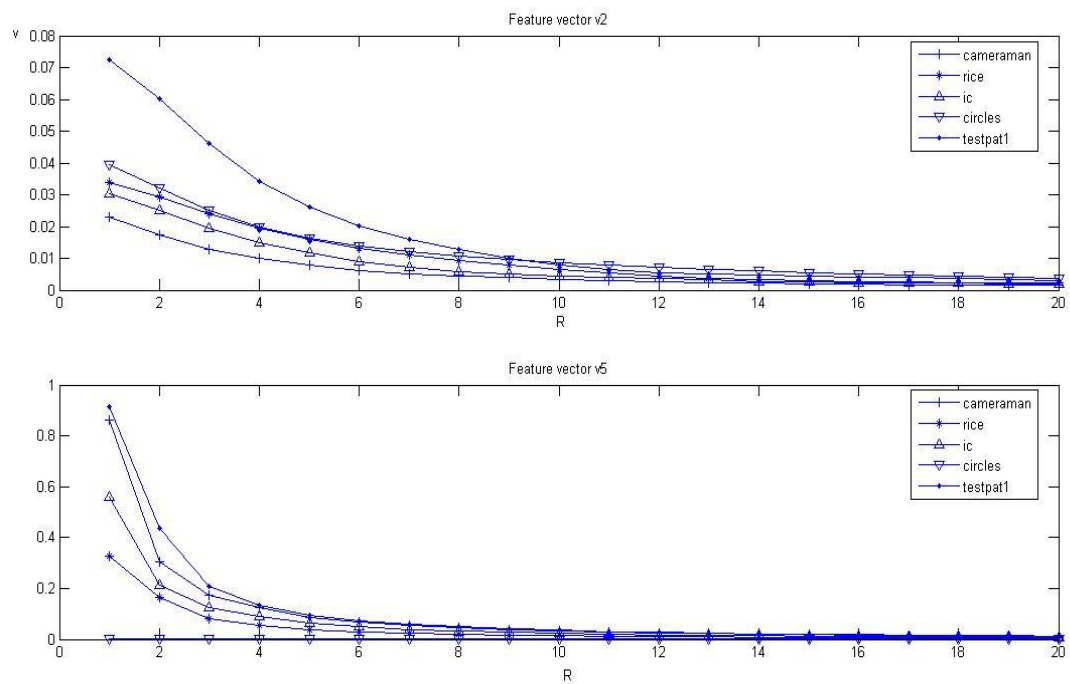

Figure 2.Curve $v_{2}$, and $v_{5}$ of Different Images

In order to estimate defocus parameter $R$, only known the roughly similar relationship is not enough. As shown in Fig. 2, every image has monotonous curve between $v_{2}, v_{5}$ and $R$, but they are not superposition. For a degraded unknown $P S F$ image, $R$ cannot be calculated because the curve of the given image is not known. For example, if $v_{2}$ of image "rice" has been calculated, and then we estimate $R$ according curve if "ic" in Fig. 2, wrong results are obtained obviously. To solve this problem, one of the methods is to choose neural networks. Computational artificial neural networks are known to have the capability for performing complex mappings between input and output data, but neural network method has bad generalization ability. Here we propose a $M G A M$ to estimate $R$ by using the variables $v_{1-5}$.

\section{TRAINING MULTIVARIATE GENERALIZED ADDITIVE MODE}

Multivariate local polynomial model is an attractive method both from theoretical and practical point of view. In this kind of multiple linear regression model, although generalizations of most of the univariate smoothing techniques to multivariate surface smoothing appear to be feasible, there is a serious problem arising: the so-called curse of dimensionality. Because of the curse of dimensionality, surface smoothing techniques are in practice not very useful when there are more than two or three predictor variables. $M G A M$ is one of good multivariate dimensionality reduction techniques. In this Section, we briefly outline and review the idea of the extension of $M G A M$ to the defocused parameter.

Suppose that the input vector is $\underline{V}=\left(v_{1}, v_{2}, v_{3}, v_{4}, v_{5}\right)$. The model is fitted by the function $R=m(\underline{V})$.

Our purpose is to obtain the estimation $\hat{R}=\hat{m}(\underline{V})$ of function $m$. This paper, we use the generalized additive model function $m(\underline{V})$ to predict the defocused parameter $R_{T}$ value based on the point $\underline{V_{T}}$ of the test image.

Consider the general multiple regression model

$R=m(\underline{V})+\varepsilon$,

where $E(\varepsilon)=0 ; \operatorname{Var}(\varepsilon)=\sigma^{2}$ and $\varepsilon$ is independent of the vector of covariates $\underline{V}$. In linear multiple regression model the regression function $m(\cdot)$ is assumed to be linear and hence additive in the predictors. In additive models the linearity assumption is dropped and the additive feature is retained, leading to the following model

$$
R=\alpha+\sum_{j=1}^{d} f_{j}\left(v_{j}\right)+\varepsilon
$$

where $f_{1}, f_{2}, \cdots, f_{d}$ are unknown univariate functions.

The additive model (5) generalizes the linear multiple regression model with the linear function replaced by an additive sum of univariate functions. The additive model can be generalized to a generalized additive model which links the mean regression function to an additive sum of univariate funcitons:

$$
f(m(\underline{V}))=\alpha+\sum_{j=1}^{d} g_{j}\left(v_{j}\right)
$$

where $f(\cdot)$ is a known link function and 
$f_{1}, f_{2}, \cdots, f_{d}$ are unknown univariate functions.

We start with describing the algorithm for fitting a parametric generalized linear model with canonical link function $f$ :

$$
f\{m(\underline{V})\}=\underline{V}^{T} \beta=\theta(\underline{V}) .
$$

Twenty original images are chosen to train the model which can be given the good relationship between them. The defocused parameter $R$ based on the digital image is the model output. The images are defocused artificially with $R$ whose value ranging from 2 to 7 . So the total numbers of training samples are 120. Then feature vectors are constructed using variables $v_{1-5}$ of each image:

$$
\underline{V}=\left(v_{1}, v_{2}, v_{3}, v_{4}, v_{5}\right) .
$$

\section{INVERSE FILTERING}

In this section, we introduce the inverse filtering approaches to image restoration that utilize minimum mean square error as an optimization criterion. The image degradation process is often represented in terms of a matrix-vector formulation of Equation (1). This is given by $y=H f+n$, where $y, f$, and $n$ are the observed, original, and noise images, ordered lexicographically by stacking either the rows or the columns of each image into a vector. When utilizing the stationary model, $H$ becomes a block-Toeplitz matrix representing the linear convolution operator $h(i, j)$. Toeplitz, and block-Toeplitz matrices have special "banded" properties which make their use desirable for representing linear space-invariant operators. By padding $y$ and $f$ appropriately with zeros so that the results of linear and circular convolution are the same, $H$ becomes a block circulant matrix. This special matrix structure has the form

$$
H=\left(\begin{array}{cccc}
H(0) & H(N-1) & \cdots & H(1) \\
H(1) & H(0) & \cdots & H(2) \\
\cdots & \cdots & \cdots & \cdots \\
H(N-1) & H(N-2) & \cdots & H(0)
\end{array}\right),
$$

where each sub-matrix $H(i)$ is itself a circulant matrix.

Notice that each block-row of $H$ and each row of $H(i))$ is a circular shift of the prior block-row or row, respectively. Classical direct approaches to solving Equation (1) have dealt with finding an estimate $\hat{f}$ which minimizes the norm $\|y-H \hat{f}\|$, thus providing a least squares fit to the data. This leads directly to the generalized inverse filtering, which is given by the solution to $\left(H^{T} H\right) \hat{f}=H^{T} y$.

The critical issue that arises in this approach is that noise amplification. This is due to the fact that spectral properties of the noise are not taken into account. In order to examine this, consider the case when $H$ (and, therefore, $H^{T}$ ) is block circulant, as described above. Such matrices can be diagonalized with the use of 2-D Discrete Fourier Transform ( $D F T$ ). This is because the eigenvalues of a block circulant matrix are the 2-D discrete Fourier coefficients of the impulse response of the degradation system which is used in uniquely defining $H$, and the eigenvectors are the complex exponential basis of this transform. In matrix form, this relationship can be expressed by

$$
H=W h W^{-1},
$$

where $h$ is a diagonal matrix comprising the 2-D $D F T$ coefficients of $h(i, j)$, and $W^{-1}$ is a matrix containing the components of the complex exponential basis functions of the 2-D $D F T$. Pre-multiplication of both sides of Equation(7) by $W^{-1}$, the solution can be written in the discrete frequency domain as $\hat{F}(\omega)=h^{*}(\omega) Y(\omega) /|h(\omega)|^{2}$, where $\hat{F}(\omega)$; $h(\omega)$, and $Y(\omega)$ denote the $D F T$ of the restored image, $\hat{f}(i, j)$, the PSF, $h(i, j)$, and the observed image, $y(i, j)$, as a function of 2-D discrete frequency index $\omega$, where $\omega=\left(k_{1}, k_{2}\right)$ for $k_{1}=0,1, \cdots, M-1, \quad k_{2}=0,1, \cdots, N-1$, for an $M \times N$ point $D F T$, and * denotes complex conjugate.

\section{SIMULATION RESULTS}

The performance of the proposed image restoration algorithm based on multivariate generalized additive model and inverse filtering has been evaluated using the classical gray-scale Moon image, Coins image, Saturn image, and Cameraman image in Matlab toolbox, and one real blurred image is used for the deconvolution procedure. The results show our method is very successful for the restoration based on this kind of blurred image. In image restoration studies, the degradation modelled by blurring and additive noise is referred to in terms of the metric blurred signal-to-noise ratio (BSNR). This metric for a zero-mean $M \times N$ image is given by 


$$
B S N R=10 \log _{10}\left\{\frac{\frac{1}{M N} \sum_{m=1}^{M} \sum_{n=1}^{N} z^{2}(m, n)}{\sigma_{v}^{2}}\right\},
$$

where $z(m, n)$ is the noise free blurred image and $\sigma_{v}^{2}$ is the additive noise variance. For the purpose of objectively testing the performance of linear image restoration algorithms, the improvement in signal-to-noise ratio (ISNR) is often used. ISNR is defined as

$$
I S N R=10 \log _{10}\left\{\frac{\sum_{m=1}^{M} \sum_{n=1}^{N}[f(m, n)-y(m, n)]^{2}}{\sum_{m=1}^{M} \sum_{n=1}^{N}[f(m, n)-\hat{f}(m, n)]^{2}}\right\},
$$

where $f(m, n)$ and $y(m, n)$ are the original and degraded image pixel intensity values and $\hat{f}(m, n)$ is the restored true image pixel intensity value. ISNR cannot be used when the true image is unknown, but it can be used to compare different methods in simulations when the true image is known.

In order to find good performances of the proposed multivariate generalized additive model ( $M G A M$ ) and inverse filtering compared with the RBF neural network algorithm (RBFNN) for defocused digital image restoration which is investigated in the literature [11], the same defocused blurred images are used for the experiments. Mean squared prediction errors are shown in Table 1. From Table 1, we can conclude that the prediction results of $M G A M$ predictor are significantly better than the RBFNN in the same simulated data.

Figs. 3, 4, 5 and 6, in which the true images, blurred images and estimated true images are depicted in the left, middle and right column, respectively, illustrate how the method behaves in Moon, Coins, Saturn and Cameraman digital images. It is Clear from Figs.3-6 that performances of the new novel digital defocused restoration method is effective in different images. Fig. 7 also shows that the presented algorithm is good for real blurred image.

Table 1. MSE using both methods

\begin{tabular}{|l|l|l|l|l|l|}
\hline $\begin{array}{l}\text { trai } \\
\text { nin } \\
\text { g } \\
\text { ima } \\
\text { ge }\end{array}$ & method & eMSE & $\begin{array}{l}\text { trainin } \\
\mathrm{g} \\
\text { image }\end{array}$ & method & eMSE \\
\hline $\begin{array}{l}\text { Mo } \\
\text { on }\end{array}$ & RBFNN & $4.81 \times 10^{-6}$ & Coins & $\begin{array}{l}R B F N \\
N\end{array}$ & $5.06 \times 10^{-6}$ \\
\hline $\begin{array}{l}\text { Mo } \\
\text { on }\end{array}$ & MGAM & $4.11 \times 10^{-8}$ & Coins & $\begin{array}{l}\text { MGA } \\
\text { M }\end{array}$ & $3.95 \times 10^{-8}$ \\
\hline $\begin{array}{l}\text { Sat } \\
\text { urn }\end{array}$ & RBFNN & $6.62 \times 10^{-6}$ & Tire & $\begin{array}{l}R B F N \\
N\end{array}$ & $8.04 \times 10^{-6}$ \\
\hline $\begin{array}{l}\text { Sat } \\
\text { urn }\end{array}$ & MGAM & $5.63 \times 10^{-9}$ & Tire & $\begin{array}{l}\text { MGA } \\
\text { M }\end{array}$ & $7.17 \times 10^{-8}$ \\
\hline
\end{tabular}
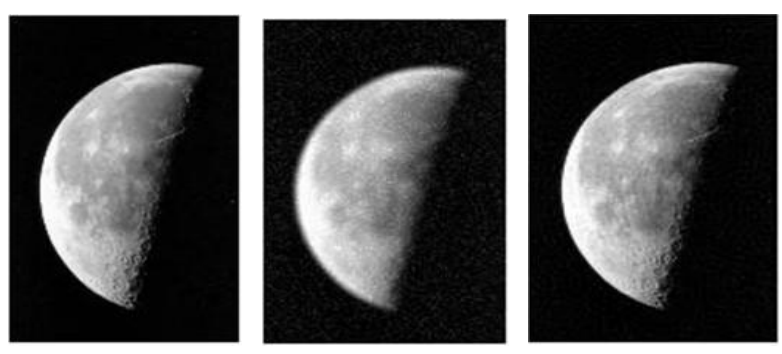

Figure.3. True image (left); blurred image (middle); estimated image (right), BSNR=12.33, ISNR=22.51
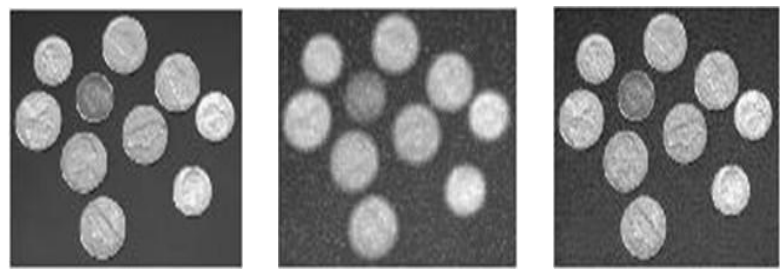

Figure 4.True image (left); blurred image (middle); estimated image (right). $\mathrm{BSNR}=11.21, \mathrm{ISNR}=23.12$
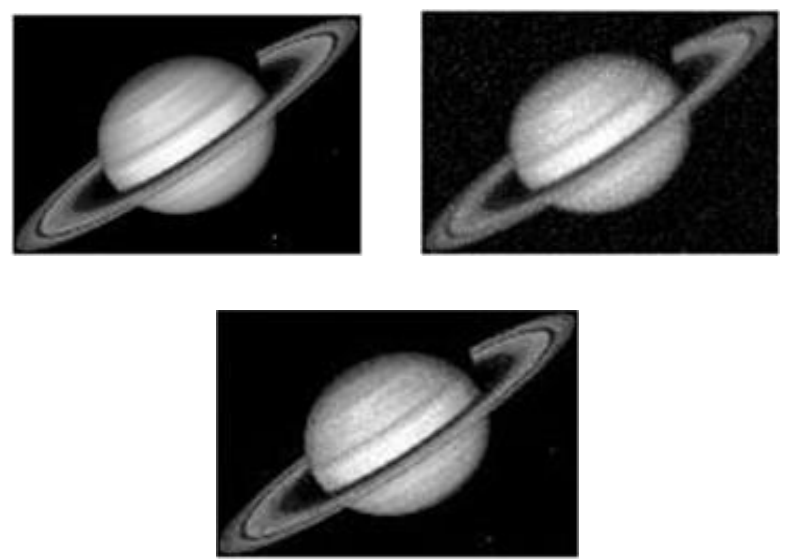

Figure 5.True image (left); blurred image (middle); estimated image (right). $\mathrm{BSNR}=13.15, \mathrm{ISNR}=24.30$
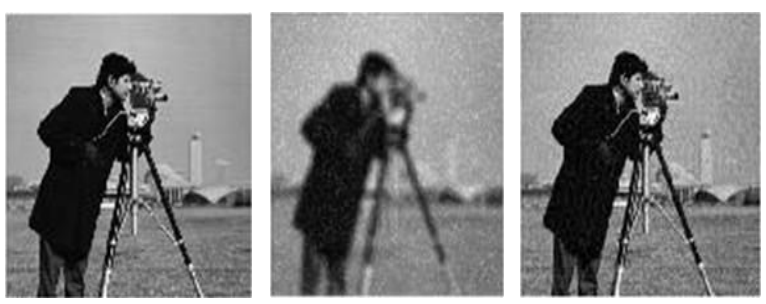

Figure.6. True image (left); blurred image (middle); estimated image (right). $\mathrm{BSNR}=11.53$, ISNR=22.07
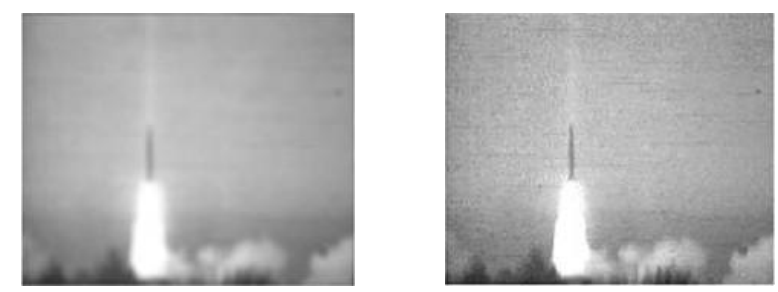

Figure.7. Result for real blurred image. Blurred image (left); restored image (right)

\section{CONCLUSION}

A new method that is based on multivariate 
generalized additive model which is a nonparametric statistical method for good high-dimensional data and inverse filtering for semi-blind restoration of blurred noisy images was proposed in this paper. Defocused digital image parameter was estimated by a multivariate generalized additive model trained in wavelet domain of the defocused noisy digital image. The main advantages of the proposed technique are that it is not only robust to noise because wavelet transform has an excellent de-noising ability, but also effective to artificially defocused and real blurred image. Defocused image restoration is successfully realized by the inverse filtering, resulting in improved the image quality. The presented algorithm was justified via simulated and practical image. Defocused image parameter can be successfully estimated by using trained model with a hybrid restoration method by using multivariate generalized nonparametric statistical additive regression model and inverse filtering. Simulated and real defocused digital image restoration results show the proposed algorithm is reliable, effective, and robust for defocused blurred noisy image restoration. Future works can be done in many fields include super resolution reconstruction, digital image interpolation, non-uniform noise, and so on. Moreover, it can be extended to other fields such as information hiding and digital covert communications.

\section{ACKNOWLEDGMENTS}

This work was partly supported by Natural Science Foundation Projects of Chongqing CSTC of China (CSTC2010BB2310, CSTC2011jjA40033, CSTC2012jjA00037), Chongqing CMEC Foundations of China (KJ120829).

\section{REFERENCES}

[1]Su Liyun, Liu Ruihua, Li Fenglan, and Li Jiaojun. Semi-blind deconvolution of defocused image with MCMA. International Conference on Computer Application and System Modeling, Vol, 10, August, Taiyuan, Shanxi, China, 2010, pp:45-49.

[2]Su Liyun and Li Fenglan. Deconvolution of Defocused Image with Multivariate Local Polynomial Regresionand Iterative Wiener Filtering in DWT domain. Mathematical problems in Engineering, Vol. 2010, 2010, pp: 1-14.

[3]Su Liyun, Ma Hong, Li Zheng, Ju Shenggen. Blind image restoration based on constant modulus with averaging and ANFIS. Fourth International Conference on Image andGraphics, 2007, pp: 143-148.

[4]Li Zheng, Su Liyun, Ju Shenggen, Yang Jian. Semi-blind Defocused Image Restoration using BP Neural Network and Inverse Filter in Wavelet Domain. Journal of Sichuan University (Natural Science Edition), 44(1), 2007, pp: 47-53.

[5]Yehong Liao, Xueyin Lin. Blind Image Restoration with Eigen-Face Subspace. IEEE Trans. on Image
Processing, 14(11), 2005, pp: 1766-1772.

[6]M. M. Chang, A. M. Tekalp, A. T. Erdem. Blur Identification using the Bi-Spectrum. IEEE Trans. On Image Processing, 39(10), pp: 2323-2325, 1991.

[7]R. L. Lagendijk, J. Biemond, B. E. Boekee. Identification and Restoration of Noisy Blurred Images using the Expectation-Maximization Algorithm. IEEE Trans. on Acoustics, Speech, Signal Processing, 1990, 38(7): 1180-1191.

[8]D. Kundur, D. Hatzinakos. A Novel Blind Deconvolution Scheme for Image Restoration using Recursive Filtering. IEEE Trans. on Signal Processing, 1998, 46(2): 375-390.

[9]A. K. Katsaggelos, K. T. Lay. Maximum Likelihood Blur Identification and Image Restoration using the EM Algorithm. IEEE Trans. on Signal processing, 1991, 39(3): :729-733.

[10]Kantz H and Schreiber T. Nonlinear Time Series Analysis Cambridge University Press, 1997.

[11]Su Liyun, Li Fenglan, Xu Feng, Liu Yuran. Defocused Image Restoration Using RBF Network and Iterative Wiener Filter in Wavelet Domain.2008 International congress on image and signal processing, Sanya Hainan, China, 2008,3: 311-315.

[12]Fan J. and I. Gijbels, Local polynomial modelling and its applications, Chapman and Hall, 1996.

[13]Su Liyun. Prediction of multivariate chaotic time series with local polynomial fitting, Computers \& Mathematics with Applications, 2010, 59(2):737-744.

[14]Su Li-yun, Ma Yan-jun, Li Jiao-jun, Application of local polynomial estimation in suppressing strong chaotic noise. Chinese Physics B. 2012, 21(2): 020508. [15]Su Liyun,Li Fenglan, Deconvolution of defocused image with multivariate local polynomial regression and iterative Wiener filtering in DWT domain. Math Probl Eng, 2010: 605241.

[16]Su Liyun, Zhao Yanyong, and Yan Tianshun, Li Fenglan. Local polynomial estimation of heteroscedasticity in a multivariate linear regression model and its applications in economics, PLoS ONE, 2012, 7(9):e43719.

[17]Su Liyun, Yan Tianshun, Zhao Yanyong, Li Fenglan. Local polynomial regression solution for partial differential equations with initial and boundary values, Discrete Dynamics in Nature and Society, vol. 2012, Article ID 201678, , 2012.

[18]Su Liyun, Yan Tianshun, Zhao Yanyong, Li Fenglan.Local polynomial regression solution for differential equations with initial and boundary values, Discrete Dynamics in Nature and Society, vol. 2013, Article ID 530932, 2013.

[19]Su Liyun, Zhao Yanyong, and Yan Tianshun. Two-stage Method Based on Local Polynomial Fitting for a Linear Heteroscedastic Regression Model and Its Application in Economics, Discrete Dynamics in Nature and Society, vol. 2012, Article ID 696927, 2012.

[20]Paavo Alku. Glottal Wave analysis with Pitch Synchronous Iterative adaptive inverse filtering. Speech Communication, 11 (2-3), pp: 109-118, 1990. 
Fenglan Li was born in Sichuan province of China, in 1979. She received his B.S. degree in Sichuan Normal University in China. She is currently a postgraduate student in software engineering of College of Computer Sciences of Sichuan University in China. She is also currently a faculty member in library of Chongqing University of Technology in China. Her research interests include applied statistics and its applications in digital image processing, information processing, and literature evaluation. She has published over 10 papers including a few high-level SCI-indexed or EI-indexed journal papers.

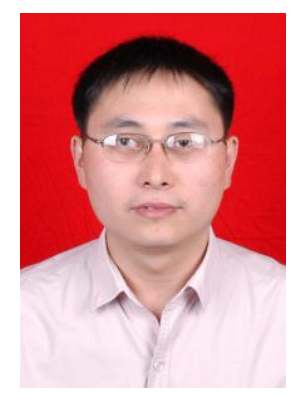

Liyun Su was born in Guang'an city, Sichuan province, Peoples' Republic of China, in December, 1977. He received the B.S. degree in applied mathematics in 2001. The M.S's and PH.D's degree were received in stochastic signal processing of probability and statistics in 2004, 2007, respectively, both from the College of Mathematics, Sichuan University, P. R. China. He is now a faculty member of school of mathematics and statistics of Chongqing University of Technology in China. His research interests include statistical signal processing, weak signal detection, digital image processing, financial statistics, and communication signal processing. He has published over 20 papers including 9 SCI-indexed journal papers and over 10 EI-indexed papers. He also is a council member of Chinese engineering probability and statistics society and Chongqing Operation Research society.

Yun Jiang was born in Chongqing of China, in 1974. She is currently a faculty member in library of Chongqing University of Technology in China. Her research interest is in digital copyright protection, information retrieval technique, and digital image processing.

Min Sun was born in Shanxi province of China, in 1987. She received his B.S. degree in Xianyang Normal University in China. She is currently a postgraduate student in School of Mathematics and Statistics of Chongqing University of Technology in China. Her research interest is in random signal processing, especially in digital image, Ultra-wideband technique, and weak signal detection. 